ainsi que de celles pour lesquelles délégation lui est donnée par le Comité. La soction permanente est présidée par. Ie conseiller d'Etat président du Comité.La répartition des affaires entre le Comité et la Section permanente est fixée par un arrêté du S.-Secrétaire d'Elal.

Un règlement d'administralion publique déterminera. les conditions d'application du présent article, notamment les conditions de fonclionnement du Comité et de la section permanente, ainsi que la composition do celte seclion, qui devra comprendre sept membres.

\section{Article 3o}

Les décrets portant règlement d'administralion publique, les décrets approuvant une concession ou accordant une autorisation, ainsi que tous autres pris en application de la présentế loi, seront rendus sur le rapport et sous le contre-seing du Ministre Président diu Conscil. Les décrets qui approuvent une concession comportant une subvention ou une avance de l'Etat, seront, en outre, contresignés par le Ministre des Finances.

\section{TITRE VIT}

\section{Article 3r}

Sonl, abrogées toutes les dispostions contraires à la présente loi Fait à Paris, Ie 24 juillet $19 \mathrm{r} 7$.

Signé : R. PoIncaré.

\section{COMMISSION EXTRAPARLEMENTAIRE}

$$
\text { DES FORCES HYDRAULIQUES }
$$

\section{Textes des Procès-Verbaux des Séances}

\section{SÉANCE DU 23 MAI 1917 (1're Séance)}

Présents. - M. le Président du Conseil, ministre des Affairc étrangères ; MN. KLotz, président ; Develle, Monestier, Gérard, Béraro, Lountes, Bedocce, Dariac, Dlisols, Jugr, Margaine, Léon Perrietr, Rrbeyre, Rousseau, Fériet du Longbois, Mamieu, Saldees, Dabat, Troté, Blazeix, Charmeil, Galllard, Conte, Duponterl, Commandant Canen, Bartirélemy, Tisserand, Hitier, Teissier, Périer de Ferar, Lavaud, Bouchayer, Arnauné, Petí, Lortie.

Excusés. - MM. Marc Reville, Barrut, Cordier, du Chanoy, C. LÉPINE.

La séance est ouverte à ro heures et demie.

M. le Présinent du Conseir. - Je ne vais pas vous faire un discours. Je veux seulement vous remercier d'avoir bien voulu accepter la tâche que nous vous avons confiée. Vous avez à nous rendre un service signalé : celui de mettre d'accord les adminisl.rations pour régler enfin une question des plus importantes, surtout en temps de guerre, celle de la bonne utilisation de nos forces hydrauliques.

Quand on écrira l'histoire de l'Administration française, on ne sera pas peu étonné de voir qu'après 25 ans, on n'a pas pu régler encore cette question, car, si je ne me trompe, c'est en r 894 que le premier projet a été déposé. Je ne compterai pas les projets qui se sont succédé. Les administrations, animées-des meilleures intentions; ne se connaissent pas, ne se pénètrent pas assez, et il est vraiment singulier que cette bataille se livre entre administrations au lieu de se livrer contre l'ennemi commun. Il faut aboutir, il le faut absolument. La Commission du Budget - et je l'en remercie - nous a signalé la nécessité de sortir de ces contradictions, de ces tergiversations : elle a demandé au Gouvernement de mettre les minislères d'accord. J'ai l'honneur de demander à M. Klotz de présider la Commission qui se réunit aujourd'hui, en raiṣon de son expérience. Je lui confie les travaux de celte Commission : il saura les faire aboutir. Je suis convaincu qu'en peu de jours vous aurez résolu le problème qui vous est soumis ; vous écouterez les raisons présenlíes de part et d'autre, vous les piserez et vous trancherez la question. L'intenlion du Gonvernement est de s'en rapporter à vous : vous trancherez la question. Nous recevrons de vos mains un projet de loi et nous le soutiendrons devant les Chambres. En outre, rous demanderons aux Administrations diverses, lorsque le projet de loi aura été établi, qu'elles auront toules pu défentre leurs travaux particuliers, d'onblier tout ce qui se sera passé jci. ll faudra l'unité complète. Ce ne sera plus le projet de tel ou tel ministère : mais le projet du Gouvernement.

Voilà l'œuvre que vous avez à accomplir : vous voyez combien clle est importante, urgente.

Je n'ai pas un seul mot à ajouter et je donne la prósidence à M. Klolz qui va diriger vos travaux avec toute sa compélence. I'espère que vous aboutirez vite el je vous remercio du service que vous aurez rendu au Gouvernement el à la République.

(M. le Président du Conseil se retire)

M. KLotz. - J'ai à vous faire connaître les excuses d'un certain nombre de membres : MM. Réville, Condier, Lépine, Barrut, du Chancy.

Messieurs, je tiens d'abord à remercier très vivement M. lo Prísident $d u$ Conseil. Je n'ai pas voulu le retenir : il a d'autres occupations. Le Gouvernement a eu la penséc de nous confier le soin de mettre au point une question délicate : comme nous voulons qu'elle aboutisse vite, je ne rous ferai pas de discours, mais je vous proposerai immédiatement une méthode de travail.

J'espère que nous serons rapidement d'accord sur cetle míthode de travail car, ainsi que l'a dit M. le Président du Conseil, après 23 années d'efforts vains et, surtont, après 34 mois do guerre, nous ne pouvons pas ici perdre un instanl. Jusqu'à maintenant, c'était le Gouvernement qui avait la responsabilité do l'inaction ; à partir de la minule présenle, c'est nous qui aurons la responsabilité si nous n'aboutissons pas. Il faut le dire. Nous sommes prêts à remplir notre devoir vis-à-vis du pays. Il n'esl pas possible, étant donnée la crise actuclle du charbon, que des compétitions diverses, que des amours-propres d'administrations prévalent contre l'intérêt général. II faut donc aboutir et, une fois que nous aurons abouti, nous avons la parole du Gouvernement qu'il ne sera plus question des revendications administratives ef que le Gouvernement présentera et appuiera le projet qui sera soumis aux Chambres, lesquelles conserveront, d'ailleurs, leur liberté enlière d'appréciation et de jugement.

Voici donc ce que je vous propose :

Les administrations des travaux publics, de l'agriculture, de l'armement et du commerce ont, loutes les qualie préparé un projet de loi. Elles sont, en réalité, les unes et les autres "demanderesses $\%$ si vous me permettez celte expression. Par conséquent, la première chose à faire serait d'entiendre un représentant de chacunc de ces administrations, exactoment comme si nous étions un tribunal. Ce délégué ferait valoir devant nous dans un exposé relativoment bref — une heure peut y suffire - les arguments principaux que l'Administration a à présenter pour réclamer le ratlachement du service. Nous n'interromprons pas les honorables représenlants des administrations. Nous les écouterons comme des juges. Lorsque les quatre administrations demanderesses auront terminé, nous prierons, si vous le voulez bien, le secrétaire de la Commission. M. l'Inspecteur des Finances Pelit. de se charger du rôle du ministère public, c'est-à-dire de formuler des conclusions et, sur ces conclusions, nous mettrons l'affaire en délibéré.

Telle est la méthode de travail que je me pormels de proposer : entendre les représentants des administrations inféressées, puis un représentant de l'Administration des Finances, qui semble tout qualifié - puisque son Ministìre à la charge de la richesse nationale - pour apporter des conclusions. Apris que nous aurons délibéré, nous désignerons un ou plusieurs de nos collegues pour rédiger, dans les quelques jours qui suivront, un texte que nous reviserons ensuite d'une façon définitive.

Nous aurons à tenir de nombreuses. séances consécutives. Nous ne devons pas nous remettre de semaine, en semaine, mais avoir 
fini dans une quinzaine de jours. Sinon, si nous ne pouvons pas prendre sur nous de siéger sans discontinuer, disons tout de suite au Président du Conseil que la tâche esl au-dessus de nos forces.

Nous pourrions déterminer aujourd'hui les jours et heures de nos séances et décider de ne pas nous livrer à de trop grands développements au cours de la discussion, élant donnée la compétence de chacun des membres de la Commission. La question nous est connue et il est inutile de la reprendre chaque fois ab ovo. Je demanderai môme à un seul représentant des administrations de présenler l'historique de la question el l'exposé des législations comparées. Nous pourrions même confier au collègue représentant le "ministiere public " de nous l'exposer. Mon honorable collègue, $M$. Develle, qui a élé magistral, ne m’en voudra pas d'emprunter à la magistralure la pratique de nos séances.

Y a-t-il, conlre celle mélhode, des objections? Il faudrait que nous siégeons lrois fois par semaine, de ro $1 / 4$ à midi $x / 4$. Nos collègues, représentant les Administrations, feraient leur exposí à tour de rôle, dans l'ordre qu'ils préféreront. el M. Pelit résumerait ensuite le débat et présenterait des conclusions.

M. Monestien, - Messieurs, j'adhère de la façon la plus complete à la mél'oule que vient d'indiquer avec tant de netleté notre distingué président, M. Klotz. Je me permettrai seulement d'émettre une opinion sur le point suivant : ne conviendrait-il pas, avant d'aborder la série des travaux, de commencer par entendre - par la bouche de M. Pelit, par exemple - un résumé des divers projets de loi qui nous sont soumis, faisant ressortir leurs points communs et leurs divergences. Peul-être entendrionsnous alors avec plus de profit les roprésentants des diverses adoministrations. C'est la sculc addition que je demanderais au programme indiqué par M. le Président.

M. Benthelemy. - J'adhère également, de la façon la plus complèle, à la procédurc proposéc par notre éminent président.

I] me permellra de lui demander un simple éclaircissement sur un détail.

Je me suis trouvé le collaboraleur des différents ministères successils qui se sont occupés de la question. Il y a 77 ans qu'on me convoque dans lontes les Commissions où il est question de la houille blanche. I'ai trouvé, dans tous les ministères, la même bonne volonté de mettre sur pied un projet de loi. Dans chaque ministère, les mèmes jdées sont revenues et ont été discutées. Par conséquenl, il y a beancoup de choses communes dans tous ces projets, beaucoup de points semblables, mais un point m'inquiète dans l'exposé que nous a fait M. le Président. Il a parlé d'une discussion qui devra porter sur la question de savoir à quel ministère il faudra rattacher la question de la houille blanche. $\Lambda$ aucun ministère. C'est une affaire interministérielle ; c'est un projet de loi à meltre sur pied aussi rapidement que possible. Qu'on n'ajoule pas une anmée de plus à ces 23 ans! Que veuton dire, en parlant de ratlachement? Ce projet de loi sera nécessaifement, comme tous ceux qui intéressent phusieurs ministères, présenté par un décret contresigné par différenls ministres. Gela n'implique pas que la question de la houille blanche sera ratlachór plulôt à un ministère qu'à un autre? S'agit-il simplement de réunir los représentants des différents ministères pour les meltire d'accord sur l'blaboration du projet?

M. Le Prf́siednt. - C'est cela I Mais il faut commencer par avoir l'exposé de chacun des textes par l'Administration qui l'a rédigć. Le Ministère des Travaux Publics nous dit par exemple, " voila ma conception ". Nous l'écoutons aflentivement. Nous entendons ensuite les autres représentants et, enfin, M. Petit nous montire les points sur lesquels il y a accord entre adminitrations, ou des désacconds de pure forme, ou des désaccords fondamentaux. Nous considérons tout de suite comme acquis les points d'accord; nous réscrvons les seconds qui pourront s'arranger et nous tranchons la question des désaccords fondamentaux. Il y a un désacord fondamental qui apparait déjà : puisqu'il y a quatre projets, c'est que chaque administration veut avoir la direction générale.

M. Dabat. - Non 1

M. le Présinent. - Mienx vaut réserver la question jusqu'après avoir entendu les quatre représentants des ministères intéressés et M. Petit qui dressera la liste des questions de principe que nous aurons à trancher.

M. Benthelemy. - Cela me donne satisfaction.

M. Le Président. - Cela doit également donner satisfaction à M. Moṇestier.

M. Bentheleny. - On va fatalement aboutir à une fusion des projets des divers ministères. D'ailleurs, les projets présentés depuis 17 ans différaient surtout parce que les époques auxquelles ils étaient préparés différaient. La question a changé constamment et, à chaque nouvelle discussion, à intervalles de deux ou trois ans, nous avons constaté qu'on avait rallié à certaines opinions, des timides que certains points effrayaient auparavant.

M. Terssier. - M. le Président, j'accepte la procédure que vous avez indiquée, mais il me semble qu’il serait nécessaịre, pour suivre avec profit l'exposé des administrations, qu'on nous distribuât auparavant les projets pour que nous puissions' y réfléchir. On ne peut pas suivre une discussion aussi grave, autssi importante, sans être très documenté, et nous gagnerions du lemps el éviterions des questions inutiles...

M. Le Présinent. - Peul-on faire distribuer ces documents ? MM. Mahien et Blazeix promettent la distribution immédiate ; MM. Dabal et le représentant de l'Armement enverront leurs documents pour la fin de la semaine).

M. le Président. - Nous commencerons donc par entendre les exposés de M. Mahieu et de M. Blazeix, lors de la prochaine séance.

M. Marreu. - Il y a aujound'hui quatre industriels qui sont absents.

M. te PrÉsinent. - Je le regrette, mais nous ne pouvons pas attendre. Nous admettrons volontiers que les industriels puissent se faire représenter par d'autres membres de leur Chambre syndicale. Nous demanderons à M. le Secrétaire Général de le leur faire savoir pour que les industriels empêchés puissent se faire remplacer, mais nous ne pouvons pas nous arrêter.

(Après une courte discussion, on décide de fixer les séances aux jours suivants : mardis, jeudis et samedis, à ro h. $\mathrm{r} / 4$.

M. Léon Perrier. -- Il conviendrait d'envisager que les membres du Parlement ayant pour la plupart à présider des Cormmissions départementales de ravitaillement, il leur sera difficile de venir le samedi.

M. LE Président. - Considérez que nos travaux ne dureront pas longtemps. Nous maintenons donc les mardis, jeudis et samedis.

M. Génnnd. - M. le Président, j'étais venu avec l'intention de donner ma démission de membre de la Commission et la discussion qui a eu lieu me confirme dans cette idée. J'ai donc l'honneur de vous remettre ma démission.

Je vois, dans la nomination de cette Commission, un déplacement de responsabilité. J'estime que, si un ministère avait bien voulu prendre en main la question, avec les lois existantes, il avait de quoi résondre le problème ! La question qui nous est posée met en jeu la responsabilité ministérielle et il serait contraire à mes i.dées de participer à des travaux qui la supprimeraient. J'ai donc l'honneur de vous remettre ma démission, tout en regreltant de ne pouvoir faire partie de cette Commission.

M. LF Président. - J'insiste vivement auprès de M. Grérard pour qu'il retire sa démission. Il ne s'agit pas du tout d'irresponsabilité ministérielle. On nous demande un service. A nous de le rendre ou de ne pas le rendre ! En temps de guerre, j'estime - c'est mon sentiment personnel - que l'on n'a pas le droit de refuser son concours au Gowvernement quand il fait appel a votre dóvouement. Aussi, mon cher ami, connaissant votre patriotisme, je vous demande de vouloir bien rester avec nous. II pent y avoir une part de vérité dans ce que vous dites : mais je suis convaincu que nos travaux pourront aboutir à un résultat et que nous aurons l'avantage de faire, nous. che que les Gouvernements n'ont pas semblé pouvoir faire, et ce ne sera pas un mince mérite.

En tout cas, mon cher ami, je n'ai pas qualité pour recevoir votre démission. C'est la Présidence du Conseil qui a constitué Ia Commission; c'est au Président du Conseil qu'il faudrait la remettre. 
M. Bedouce. - C'est exactement pour les raisons que vous venez d'indiquer que la Commission des Travaux Publics de la Chambre a demandé la constitution de la présente Commission ; c'est parce que nous nous rendions compte que la lutte entre Ies Administrations aboutissait à l'absence de résultats que nous avons insisle pour qu'on aboutisse rapidement par une Commission qui servirait d'arbitre. Nous sommes des conseillers choisis par le Président du Conseil qui, lui-même, arbitrera nos décisions.

M. Margaine. - Je me permels d'insister auprès de nolre collègue, M. Gérard, pour qu'il veuille bien ne pas se retirer de la Commission. Nous, Parlementaires, nous sommes profondément affectés par la praralysie qui atteint le Gouvernement sur ces questions depuis si longtemps. Mais, c'est justement à nous, Parlementaires, à ne pas nous retirer quand, en proie à cette paralysie, il essaie d'en sortir par notre concours! Qu'il soit fâcheux qu'il ait à employer ce moyen, je suis le premier à le dire, mais, quant à se retirer, je vous demande de ne pas donner cet exemple.

M. Gérard. - J'aurais compris une Commission composée de sept ou six membres.

M. Margatne. - Elle se réduira peut-être à ce nombre !

M. Le Président. - Je ne trouve pas que nous soyons une Commission trop nombreuse ; car il y a beaucoup d'intérêts en cause. Je crois que nous aboutirons, parce que nous avons le sentiment du devoir à remplir. Il faut aboutir.

M. Margaine. - Nous ne pouvons pas ne pas aboutir!

M. l.e Président. -- Ft je demande à M. Gérard de rester avec nous...

M. Géraro. - Alors, je reste.

M. t.e Président. - Je vous remercie. L'incident est clos.

M. Benoucr. - Nous. essaierons de mettre en main un programme de moyens d'exécution qui mettront d'accord les différents départements.

M. Monestier. - Cela laisse les responsabilités antérieures.

M. Dabat. - Monsieur le Président, on met toujours les Administrations en cause. Je voudrais faire remarquer que, depuis Ino2, le Ministère de l'Agriculture a déposé un projet de loi qui n'a pas élé voté par les Chambres. De son côté, le Ministère des

"vaux Publics a déposé il y a quelques années un projet de loi qui est allé jusqu’au Sínat. Nous ne sommes pas tout à fait fautifs.

M. cr Présintrar: - Nours nouvrons pas le debat sur la ques-

$n$ des responsabilités. Nous sommes ici pour aboutir, je le répc̀le. Ce que ie demande aux Administrations, c'est qu'après me nous aurons dit "voilà le projet qui convient", aucune ne s'avise, par des procédés qui sont quelquefois dans les habitudes de certains, de contrecarrer les décisions de la majorité de cette ammission. Une fois que le Président du Conseil, que le Gouyornement se sera rallié à nos vies. il faut qu'il soit bien entendu que toutes les A.dministrations s'inclineront. Nous ne nous occupons plus du passé : nous avons une hesogne positive à accomplir

non une besogne négative. Nous laissons à d'autres la critique.

Et, comme rous sommes tous des hommes occupés et que nous mo nourons commencer aujound'hui à entendre un exposé quelconque, nous levons la séance en fixant à samedi matin (10 h. $\mathrm{r} / \mathrm{h}$ précises) la prochaine réunion. Une convocation sera, d'ailleurs. chaque fois adressée aux membres nar le secrétaire général.

I,a séance est levée à II h. I/h.

\section{SÉANCE DU SAMEDI 26 MAI 1917 (2e Séance)}

La séance est ouverte à ro h. I/4.

Excusés : MM. Léon Perrier, Révillee, Rousseau, Cordier, nu Chanoy, Lépine, Barrat.

M. Le Préstdent. - Messieurs, la séance est ouverte.

Vous vous rappelez ce que nous avions décidé : que des suppléants pourraient être désignés pour remplacer les industriels qui ne pourraient assister à nos réunions. Ces suppléants ont été désignés : ce sont MM. Pinot, Marlio, Botganlt. Tous trois, je rois, sont présents aujourdhui et je les remercie de vouloir bien participer à nos travaux.
La parole est à M. Mahieu au nom du Ministère des Travaux Publics.

M. Mabieu. - Le problème pour la solution duquel nous sommes réunis est à la fois très ardu et très simple. Il est ardu parce cu'il faul concilier des inférêts considérables, très simple varce que, logiquement, il ne peut être résolu que par l'unité 'e conception, l'unité de direction el l'unité de responsabilité dans la rcchenche des moyens de tirer le meilleur parti de l'énergie contenue dans nos cours d'eau.

Jusqu'en r $88 \mathrm{r}$, les questions concernant les cours d'eau de toute nature ressortissaient au même Ministère des Travanx Publics, du Commerce et de l'Agriculture, et étaient étudiées par' ses seuls services.

Quand on décida de créer un Ministère spécial de l'agriculture, ce dernier revendiqua la gestion des cours d'ean non navigables ni flottables, parce que, disait-il, destinés spécialement of presque uniquement à satisfaire des besoins a gricoles. Et, en effet, on peut dire qu'à oe moment, personne ne songeait à capter l'énergie mécanique contenue dans les cours d'enu pour en tirer un parti nettement industriel et en faire le commerce. Ce ne fut qu’à la fin du siècle dernier que l'industrie hydraulique prit son essor et elle a fait, depuis, des progrès immenses. Quoi qu'il en soit, en $\mathrm{r} 88 \mathrm{x}$, la thèse de l'agriculture se défendait et le Gouvernement s'inclina. Il n'y ent qu'une seule personne pour pro'ester, ce fut M. Alfred Picard, qui ent la prescience de ce que pourrait donner l'énergie des cours d'eau. Il avait demandé, au moins, que, pour les grands travaux d'aménagement des eaux, leur étude et leur exécution fussent partagées entre les Travaux 'ublics ef l'Agriculture, suivant qu'elles seraient plus particulirement destinées à la réalisation de l'amélioration du régime des 'ux ou d'améliorations agricoles. Ricn n'y fit et ,pourtant, si on 'avait suivi, nous ne serions pas ici pour chercher à rélablir

' wité détruite en I88I. En fait, depuis la période s'étendant do

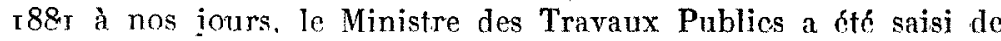
'ms les projets d'usine à établir sur les cours d'ean du domaine niblic et le Ministre de l'Agriculture sur toutes les autres.

Jusqu'en rgo/4, en matière d'usines établies sur les cours d'enu du domaine public. toutes étaient soumises an régime de l'autorisation ; en principe, cette autorisation donnée par décret sur la pronosition du Ministre des Travaux Publics, aprìs avis du ronceil d'Etat, est précaire et révocable, mais, en fait. elle est nermanente, ot on ne connait pas d'exemple de rérocation. Toutefois, un certain nombre d'usines étaient concédées, mais comme accessoires d'autres entreprises déclarées d'utilité nublique tellos qué : chemins de fer, canaux, etc. En rñ 4 . le Ministre des Trnvaux Publics a fait admettre par le Conseil d'Etat m'il powvait désormais réglementer nar voie de concessions de travaux nublics. les usines produisant de l'énergie nour la disfribuer et en fairc le commerce, toutes les fois qu'elles desserviraient nlusieurs éta-

':aements publics ou privés. Le commerce de l'énergie est donc devenu une industrie qui a sa vie propre, indénendante de celle des antres industries ani en fontt usage et. des lors, on a nu lui annliquer les principes généraux concernant les couvres d'ntilitó nublique. La première application de rette doctrine a rtó faite par le décret du 25 octohre rarli: aui concìde l'étahlissement ".ne usine sur la Basse-Isère, à Beaumont-Monteux ef. 'désormais. pour tontes les usines dont l'objet nrincinal est le commere de l'énergie, c'est un décret de concession dans la même forme cni autorisera. Pour les autres nsines consommant elles-memes leur énergie nour l'eleotro-chimie on l'électro-métallurejie, par exemple, la loi et les règlements en vigueur laissent. subsister le régime de l'autorisation.

Tel était le systeme des travanx nublics an moment de l'onverture des hostilités, et il a fallu. rlors' l'adapter àux nécessités de la dófense nationale qui exigeait la nroduction rapide de force nnfrice. La procédure suivante a éfé adonfée d'accord entre le vinictre des Travaux publics et le Snus-Secrétaire dTtat des. Mrinitions, d'abord, le Ministre de l'Armement et le Sous-Secrétaire d'Ftat des Fabrications de Guerre. ensuite.

Les deux Administrations dressent la liste des usines à établir ou à renforcer. L'Armoment fait connaitre celles de ces usines 
qui lui paraissent nécessaires et le Ministre des Travaux Publics délivre les autorisations nécessaires. Etant donné la longueur des formalités ou des consultations indispensables pour arriver à lautorisation par décret ou à la concession, le Ministre des Travaux Publics donne, par simple arrèlé, des autorisations provisoires d'exécuter immédiatement los travaux avec les réserves tochniques ou agricoles nécessaires et à la condition que le bénéficiaire se soumettra au régime de la concession tel qu'il est institué par le décret de тوr4́ avec participalion de l'Etat aux bénéfices. Les travaux penvent ainsi s'exécuter pendant que les formalités s'accomplissent. Celle nanière de faire a l'avantage de décharger le Ministre de l'Armement te toute besogne administrative et de lui conserver le rôle d'action qui est le sien. C'est le Ministre des Travaux Publics, celui qui, dans l'avenir, doit avoir le conlrible de l'usine et qui a la gestion du cours d'eau, qui fixc les conditions de la concession ef, en particulier, établit, les bases de la parlicipation de l'Ftat aux bénéfices. Le résultat de colte orgnaisation de guerro est, d'ailleurs, des plus encourageants, et je crois pouvoir dire que jamais la Défense Nationale n'a attendu les autorisations dont elle avait besoir.

Majs, il nous faut aussj songer à l'après-guerre et à l'jmpériense nécessité où nous sommes de suppléer sans tarder, par tous les moyens, à la pénurie de houille dont notre pays souffre aujourd'hui et dont il continuera à souffrir en lemps de paix. Or, l'expérience a démontré que l'électricité pouvait aujourd'hui remplacer le charbon dans ie nombreux cas et les progrès des industries éleictro-chimiques el électro-métallurgiques réalisés pendant la guerre par des industriels bien connus dont plusieurs cont parlie de la Commission, sont tels que tous les espoirs sont permis en matière d'utilisation industrielle du courant électrique.

La France veut donc suppléer à sa pénurie de honille par un bon amónagement des ressources en force hydraulique dont elle est pourvue en abondance ; elle peut, en particulier, l'utiliser en grand pour la traction de ses chemins de fer et éviter ainsi des transporls onéreux et des importations inutiles. L'on pent, d'ailleurs, se faire une idée de l'économie de houjlle noire qu'on pourra réaliser par ce procédé si l'on constate qu'un kilowattheure équivaut en une année à une consommation de houille le 8 ou 9 tonnes selon les uns, de i 5 tonnes, suivant d'autres.

Il faut donc aménager nos cours d'ean et, pour ce faire, quatre séries d'opérations et d'études sont nécessaires :

$I^{\circ}$ On envisagera chaque cours d'eau et ses affluents dans leur ensemble et on établira le programme d'aménagement en vue de la production de l'énergie, sans tenir compte de la ligne imalginaire à ce point de vue qui sépare la partie domaniale de celle mui ne l'est pas ;

2" On étudiera en même temps la répercussion que les travaux to ce programme, supposé effectué, auront sur la navigation ef les crues, d'une part, ef sur les inlérêts agricoles et industriels. "...tre part ;

$3^{\circ}$ On réduira le progrimme dans la limite strictement imposée par les intérêts visçs alı paragraphe 2 ;

$4^{\circ}$ Enfin, on cherchera à équiper les diverses chutes ainsi indiquées.

La hesogne est rentaiuement considérahle, très difficile, et elle exige le cononurs :omplet aussi bien des Administrations que des Industriels. I! faut, pour arriver an but poursuivi, tenir compte à la fois des ronditions lechniques et économiques du problime "les lonis nrmières parties du programme sont intimement lifes : la nromière est purement technique et les trois nutres mixtes. Jamais un insustrin ani anrait a résoudre le prohlème ne penserait ì en faire óludier les diverses parties par des services différents en chargènt quelqu'un de les mettre d'accord. L'Etat, en l'espòce, doit agir comme rn industriel avisé ef. comme lui, ríalienr l'unité de vues dans ses études. Cette unité de vues pourrait-elle être assurée par une autre Administration rue le Ministère des Travaux Publics? To ne le crois pas. Le Ministère dise Travaux Publics est chargé de la gestion de la navigation et des travanx de défense contre les inondations ; it est le représentant du riverain d'aval, celui qui a la gestion do l'aval, et c'est certainement celui qui est le plus intéressé à l'amé- nagement des eaux. Les travaux qu'il fera à l'aval ne seront pas susceptibles - ou très rarement - de gêner l'amont. Au contraire, si l'on exécute des travaux à l'amont sans en référer à laval, il pourra se produire des inconvénients considérables et la répercussion que les usines peuvent avoir sur les crues ou la navigation est certaine. Je demanderai à Monsieur le Président 'c donner, sur ces points spéciaux, la parole à M. Salles, président de la Commission des annonces de crues et président de la deuxichme Section "hn Consel Général des Ponts-et-Chaussées, 'argé spécialement des questions de navigation. Il expliquera, par des faits précis, les inconvénients à craindrc.

M. LE Présment. - Je donne la parole à M. Salles, toujours au nom du Ministère des Travaux Publics.

M. Salles. - En ce qui concerne les annonces de crues, on cherche depuis longtemps des formules cmpiriques pour prévoir quelle sera, par exemple, la hauteur de la Seine à Paris, étant. donné qu'on constate telle hauteur sur tel affluent. On peut, dans une certaine mesure, indiquer quelques jours à l'avance quelle sera la hauteur de la Seine à Paris. Si on établit une usine hydraulique, ces prévisions peuvent changer. Depuis longtemps, le service des annonces de crues demande que, toutes les fois qu'on étahlit une usine, on s'entende préalablement avec l'agent chargé de l'annonce des crues. Il peut tenir compte, dans une certaine mesure de ce fait de l'homme qui est à ajouter au fait de la nature.

En particulier, si une usine située dans la partie haute de la rivière vient il lâcher ses caux sans prévenir, il en résultara pour l'aval un afflux supplémentaire qui bouleversera le régime des annonces de crues et qui pourra, dans certains cas, les augmenter notablement au grand dommage des riverains. Il y a donc nécessité d'établir des relations étroites et constantes entre le servicc les usines et le service des annonces de crues, sans cela il y aurait lies perturbalions dans ce dernier.

Je passe au point de vue de la navigation.

Lcs usines hydrauliques ne prennent pas le même volume d'eau loutes les heures du jour. Pendant la nuit, les besoins sont plus 'aibles. Lorsqu'au contrairè, les besoins de l'usine sont plus grands, il faut que l'usine prenne à la rivière, non pas sa dotation normale, mais une fois et demie ou deux fois cette dotation.

S’il n'y a pas de barrage à côté de l'usine et s'ill existe une prise d'eau directe. il n'y a pas d'inconvénient : l'usine prend à chaque instant un volume d'eau déterminé, mais le rendra immédiatement en aral de son canal de fuite sans apporter de perturbation au régime des eaux. Au contraire, s'il y a un barrage pendant 'es heures de nuit, l'usine écculera moins d'eau qu'elle n'en recevra. Elle accumulera une réserve qu'elle lâchera ensuite pendant les heures de pointe. Il en résulte que l'usine apportena; à l'aval de son barrage, une perturbation dans l'écoulement régulier des eaux de la rivière. Anx heures de nuit où elle reçoit plus qu'elle ne donne, il en résultera un abaissement du débit, un abaisse'nent de la hauteur d'eau. $\Lambda u$ contraire, aux heures où l'usine donne plus qu'elle ne recoit, quand elle restituera la réserve faite pendant, les heures fe riuit, la rivière d'aval recevra plus d'eau que la rivière d'amont, it y aura donc plus de hauteur d'eau, 'ndant ces heures; à l'aval qu'à l'amont. Supposons, dans ces condilions, qu'un hateau ayanl sa charge normale parte de Lyon, 'nr cremple, pour aller vers Beaucaire. Supposons que la hau'lur d'eau soit de I $\mathrm{m}$. 40 à l'aval. Si le bateau traverse une "ague produite par une usine siluée en amont, cette vague pourra amener le bateau à échouér parce que, au lieu de $1 \mathrm{~m}$. 40, il trouvera plus que I $\mathrm{m}$. 20 de hauteur d'eau.

Voilà l'ordre de grandeur de ces vagues qui onl été consta'es sur la rivière du Rhône, surtout après l'exécution des usines de Genève et, plus récemment. de l'usine de Jonage. En aval 'e Genève jusqu'à Lyon, la vague a 30 ou 40 centimètres. La navigation est faible dans cette partie.

Entre Lyon et Valence, la vague a encore une valeur de 20 cenרètres. A Valence, elle s'abaisse à ${ }^{2} 2 \mathrm{~cm}$. et reste à 10 jusqu'à Reaticaire, après 300 kilomètres on aval de l'usine. Ce ne sont pas des hypothèses, mais des faits concrets. 
Les vagues produites par les usines peuvent donc avoir une influence considérable sur la navigation, si on n'assure pas la : oncordance absolue entre les deux services.

Si nous prenons le cas dune usine siluée plus près de la mer, - orce de la vague n'aura pas le temps de s'atténuer en route.

Par conséquent, toutes les fois qu'on aura à étudier l'établissement d'une usine sur un cours d'eau, à sa partie supérieure ou a sa partie inférieure, il devra y avoir des conférences très sérienses avec les services de navigation pour examiner quelles seront les perturbations causées par la marche de cette usine en ce qui concerne la navigation du cours dicau. Il faudra conciliex les deux intérèts, el je crois que la seule solution capable de réduire les inconvénients au minimum est que les deux soient sous le mème contrôle.

M. LE Président. - Nous vous nemercions de cette communication.

M. Manrev. - Personne ne pent donc nier que l'élablissement de dérivations ou de barrages dans la partie supérieure d'un cours d'eau ou de ses aftiments, doive le plus souvent en modifier le régime à l'aval et, par suite, changer les conditions de navigabilité ou d'écoulement des eaux. Des conférences pourront être possibles entre services intéressés, mais il est permis de douter de leur efficacité et surtout de leur solution rapide.

En ce qui concerne les inondations en particulier, elles sont fonction des conditions d'écoulement qui leur sont assurées et le meilleur moyen de les éviter consiste à ralentir cet écoulement dans les parties supérieures ; mais il est clair qu'aucun résultat ne pourra ètne obtenu si un autre service s'efforce, dans cette section, de faire biler les eaux au plus vite. D'un autre côté, si on multiplie dans la partie haute des cours d'eau les ouvrages de retenue, si on y crée des réservoirs de crues ou d'étiage, on modifiera le débit de l'aval; rationnellement faits, ces travaux peuvent régulariser le débit de la rivière en tout temps, c'est-à dire augmenter le débil d'étiage de toutes les usines d'aval et, par suite, supprimer tout ou partie des chevaux thermiques qui viennent en basses eaux, au secours de la rivière.

Il est donc indispensable - et j'appelle sur ce point l'attention de la Commission - d'établir un plan d'aménagement général de la rivière ; c'est le seul moyen de tirer un parti complet de ses réserves d'énergie. Pour préciser, je vais vous citer un fait concret :

Le Ministre des Travaux Publics esl actuellement saisi de plusieurs projets d'aménagemnt hydraulique de la Haule-Dordogne entre Bort et Argentat. L'un de ces projets est particulièrement intéressant : il porte le débit de l'étiage de 20 à bo mètres cubes el parmettrait, par suile, d'accroître très sensiblement le nombre de chevaux dont on pourrait disposer en basses eaux sur loute la rıvière. Or, au-delà de Bort, la Dordogne se développe encore sur plus de 200 kilomètres; ellé y recoit des allluents el il est à prévoir qu'un bon aménagemenl de celte région augmentera encore le délit détiage; mais la rivière, à partir de Bort, ne fait plus parlie du domaine public. Faut-il que les éludes du Ministère ues Travaux Publics s'y arrêtenl et qu'il n'établisse, entre Bort el Argental qu'un projet incomplet parce que, au-delà de Bort, 1 aménagement de la rivière rentre daris les altributions du Ministère de l'Agriculture ou du Ministre de l'Armement si on s'en rapportait au décret de I9I6? M. Ie Ministre des Travaux P'ublics ne l'a pas pensé el nous avons continué les études sur Ionte la rivière et ses affluents. Il est clair, en effet, que si une nouvelle réglemenlation du débil d'étiage est possible par un aménagement au-delà de Bori, les usines d'aval doivent être étaolies en conséquence et il faut se hâter de résoudre le problème dans la partie haule. Nons pouvons peut-être faire ces études, mais la décision ne nous appartiendra pas et je ne peux pas m'empêcher de constater que c'est là une mauvaise condition pour réaliser l'utilisation rationnelle et complète des cours d'eau. A un autre point de vue, de par la loi du i 5 juin Igo6, c'est le Ministre des Travaux Publics qui a le contrôle des distributions d'énergie ćlectrique. Dès que le courant est créé et sort des bornes de l'usine, il est sous le contrôle du Ministre des Travaux Publics, quelle que soit sa provenance. C'est lui qui a les pouvoirs néces- saires pour donner les autorisalions, contrôler el surveiller les distributions du courant. Or, depuis les hostilités, chacun de nous sait les services immenses que se sont rendus les diverses usines en s'entr'addant muluellement. C'est ainsi que les usines de la Loire sont alimentées, en partie, par du courant venant de Grenoble et des Hautes-Alpes. Dans ces conditions, il serait certainement avantageux d'avoir des courants qui aient la même firéquence el la mème périodicité el, si ce n'est pas la même autorité qui contròle les usines el les distributions d'ćnergie, il faudra encore des conférences nombreuses pour se meltre d'accord sur la nécessité d’imposer la production de tel ou tel courant. Je ne rois pas trop comment réaliser la simplification el la rapidité que doil chercher la Commission si cetle dualité est ćtablie et je crois que c'est là une raison de plus pour dire que le Ministre des Travaux Publics est le seul qualilié pour avoir le contrôle de l'ensemble des usines hydro-électriques el de leur's distributions. Les décisions y gagneraient en rapidité, la consullation d'agents appartenant à deux administrations diflérentes devant fatalement provoquer des relards. Le Ministre des Travaux publics possède un personnel lechnique au courant des études hydrauliques; cesi lui qui lravaille pour le comple du llinistère de l'Agriculture el du Ministère de l'Armement. Donk, si on veut faire l'uniticalion, si l'unilication est reconnue désirable par la Commission, le Minislère des Travaux Publics paruî le plus qualifié pour en ètre chargé.

On pourrait ètre tenté de laisser à chacun des services existants le soin d'étudier les alfaires de son domaine, quilte à centraliser dans une même main les résultals, mais, c'est là une solution hybride, incapable à nolre avis de donner à l'induslrie hydranlique l'impulsion dont elle a besoin. Il ne sagit pas de meltre d'accond deux Administrations, mais de trouver la solution qui donnera le rendement maximum el qui supprimera le plus possible de consultations, de conlérences, elc..., e'cst-ändire de sources de retards et de conflits. Vouloir faire éludier l'aménagement rationnel d'une riviere par deux services distincts et en charger un troisième de les metlre d'accord, est faire ouvre vaine, el une pareille organisation ne pourrail aboutir qu'à la création d'un personnel spécial qui dépendrail du nouvel organisme, el qui serait chargé de l'ensemble du travail. Par la force des choses, cet organisme serait amené, sinon à trancher, du moins à peser fortement sur la solution de toutes les questions de navigalion el de défense contre les crues el l'on ne voil pas iris bien a priori comment ce ne serail pas le Ministre des Travaux Publics, plus ou moins conseillé el aidé, qui ne serait pas chargé de cetle besogne.

El, enfin, le Ministre des Travaux Publics n'est-il pas celui qu a déjà la charge des grands services de la production nalionale : llines, Chemins de fer, Navigation, Porls. N'est-ce pas lui qui gère l'outillage public de la nation el qui lournit les malières premières de l'industrie contenues dans le sous-sol du pays fill n'y a aucune raison pour ne pas lai donner la gestion de lensemble les forces hydrauliques et de séparer des autres celte source d'énergie el de richesse, surlout quand on constate qu'clle ne peut donner son rendement maximum que si elle se dévoloppe et fonctionne en plein accord avec des services du Ministère des Travaux Publics qu'il est impossible de lui enlever sans le supprimer virtuellement et le fondre dans les autres départements ministériels.

Est-ce à dire que le Ministre des Travaux Publics décidera seul, avec ses services techniques if Tel n'est pas mon avis. Aussi, dans son projel de loi a-t-il prévu l'instilulion d'un Comité des forces hydrauliques, composé, mi-partie de fonetionnaires, mipartie d'industriels, of M. lo Ministre des Travaux Publies est ltécidé à faire appel à MM. les Membres du Parlement appartenant aux grandes Comunissions qui s'occupent de la question des isines hydrauliques pour y prendre place, s'ils accoptent de pariciper à ses travaux.

C'est une institulion analogue à celle qui fonctionne depuis la loi du 13 juin rgo6, pour les distributions d'énergie. Les services rendus par celte instilution sont connus de tous et laccord est complet à ce sujet entre l'Administration ef les Industriels. 
Ce Comité serait obligatoirement consulté sur les plans d'aménagement des eaux, sur les conventions et cahiers des charges des autorisations et des concessions ; il pourrail demander les cludes et enquêtes qu'il jugerait utiles pour établir son opinion á son programme d'action. Mais, j'insiste sur ce point, il ne pourrait pas faire acte de gestion. C'est le Ministre des Travaux 'ublics, responsable devant le Parlement et devant le Pays, qui seul dolt prendre les dócisions sur avis motive du Comité.

Je passe à l'exposé rapide du projet de loi.

Le Ministre des Travaux Publics pense que le maintien du statu-quo n'est pas désirable ; la dualité d'autoritó résultant de la distinction entre cours d'eau domaniaux el non domaniaux, ne peut que nuire à l'aménagement rationnel de nos forces hydraudirfues.

Au point de vue des travaux exécutés dans une parlie quelconque de leurs cours, lleuves el rivières doivent ôtre envisagés dans leur ensemble el cela, en raison de la répercussion certaine qu'auronl, sur le régime du cours d'eau à l'aval (crues ou navigation) les ouvrages installés à l'amont.

Non seulement pour la sauvegarde de tous les intérêts en présence, mais encore pour l'utilisation la plus complebe de la force disponible, l'unité de direction, de dócision et de conbrôle est indispensable.

Enfin, dans le but, non négligeable, d'abrégrer les longueur's de la prockdure d'insiruction des demandes, il faut qu'une scule autorité stalue el tranche en dernier ressort.

\section{Régime proposé :}

Le service des forces hydrauliques est placé sous l'autorité du Minisire des Travaux Publics qui a dans ses altributions la navigation et la survejllance des crues.

Il sera procedé, d'aceord avec le Ministre de l'Agriculture el le concours du Comilé des Forces hydrauliques, à l'élablissemeni d'un plan général des caux, pạr riviêre et par bassin.

Pour moner à bien la tache qui lui est assignée, le Ministre s'adjoint, en effet, un Comité des Forces hydrauliques composé de membres des grandes Commissions du Parlement compétentes en malitre d'usines hydraulicques ef en nombre égal de fonctionnaires al d'industricls; ce Comitć serail obligatoirement consulté sur les questions générales visant l'amćnagement des cours d'eau, sur les difficullés particulières qui pourraient surgir lors de l'instruction d'une affaire et sur les projets d'autorisation el de concossion.

Pareille exoncoption a élé réalisée à l'étranger : en Norvège, en Jalie, en Suisse, et dans divers litats américains.

II reste a voir comment fonctionnent ces rouages :

A. - Aulorisations. - Elles sont accordées sur tous les cours d'eau, par les Préfets agissant sous l'aulorilé du Ministre des Travaux Publics. Ce dernier ne slahera directement que, lorsque sur les cours d'eau domaniaux, la durée de l'autorisation excédera cinq ans.

B. -- Concession. - L'instruction, selon les formes en viguenr, sauf dólais à ohserver, sera dirigée par le Ministre des Travaux Publics qui proposera, après avis des ministres intéressés, la déclaration d'utililé publique. Sur les cours d'eau non domanianx seulement of lorsque l'accord n'aura pu se faire aver le Ministre de l'Agriculure sur les intérêts agricoles, le Conseil des Ministres Iranchera.

Telle est la procódure qui permet de réaliser l'unité de direction el, assure la sauvegarde de tous les intérèts en présence et de rapides décisions.

Dans l'article premier du projet de loi, le Ministre des Travaux Publics a précisé - reprenanl lidé émisc dans le projet de loi de MN. Bedouce al Margaine - que l'énergie contenue dans les cours d'ean élail assimilable aux mines siluées dans le sous-sol des propriétés privécs, et constiluait me richesse nationale à incorporer au domaine public. Il a lenu à prociamer ce principe pour pouvoir établir les droils des particuliers, des communes, des départements ou de l'Etat.

Ce principe soulèvera certainement quelcues objections ; nous Jes disculerons le moment venu.

En ce qui concerne la pirlicipation de l'Ftat aux bénéfices, le Ministre des Travaux Publics a envisagé - comme MM. Be- douce et Margaine - plusieurs catégories de redevances ': redevance fixe, proportionnelle à la valeur localive, redevance variable, proportionnelle aux bénéficss de l'usine. Nous on examinerons les modalités plus tard.

Les usines exislantes seraienl toutes ramenées au régime de la loi dans un délai de $7^{5}$ ans à partir de sa promulgation. Nous estimons, en effel, qu'il faut laisser à chacune de ces usines soixante-quinze ans pour amortir les dépenses d'acquisition des terrains et d'installation des bâtiments et machines devant revenir graluitement à l'Etat.

Une indemnité calculée d'une façon spéciale, inciterait toutes les usines ì rentrer dans le droit commun avant soixante-quinze ans ; quand à celles qui désireraient augmenter leur puissance, capter une nouvelle source, elles ne seront autorisées à le faire qu'en se plaçant sous le régime de la concession.

Enfin, le dernier article institue le Comité des Forces hydrauliques, et son texte serail madifié par l'adjonction d'un certain nombre de membres du Parlement s'ils veulent bien accepter d'en faire partie.

Telle esi, hiessieur's, léconomie du projet du Ministre des Travaux Publics ; il assure l'unité de conception, l'unité de direction et l'unité de responsabilité nécessaires pour tirer des forces hydrauliques le maximum d'effet utile.

M. Le Président. - Le Ministère des Travaux Publics ayant terminé son exposé général, je donne la parole à M. Blazeix au nom du Ministre du Commence, de I'Industrie, des Pastes et des Télégraphes.

M. Blazeix. - (Voir la nole spécialle qui a été adressée aux membres de lia commission).

M. Le Président. — Nous avons entendu les représentants des deux ministères ; il nous resle à connaître l'exposé des Ministres de l'Agriculture et de l'Armement, et je proposerai ensuite de idemander à MM. Margaine et Bedouce ainsi qu'à la Chambre syndicale des Forices hydrauliques de nous résumer leurs propres projets.

M. Albert Gérard, sénateur, demande s'il ne semait pas possible que la Commission ait entre les mains un travail d'ensemble résumant la situation des usines anciennes qui ont la pleine propriété de leurs installations. Les projets de loi présentés les visent-ils ?

M. Maniev observe que tous les projets de statut des Usines hydrauliques soumis jusqu'ici an Parlement, de même que ceux acluels excluent les usines fondées en titre que vise M. le Sénateur Gérard.

M: Bedouce, député, voudrait que les services des Travaux Publics et de l'Agriculture fournissent à la Commission la statistique complète des usines existantes, en cours d'aménagement ou projetées.

M. Manieu déclare qu'en ce qui concerne ces renseignements statistiques, il est prèt à les faire connaître à la Commission pour les usines du domaine public, mais que pour les usines fondées en titre, un délai d'une semaine environ lui est nécessaire.

M. le Président prie M. Manine et M. Dabat de se mettre d'accord pour fournir à la Commission les éléments d'une statistique complète.

La sćance est levée à $\mathbf{I} 2$ heures.

\section{SÉANCE DU 29 MAI 1917 (3e Séance)}

Présents. - MM. Klotz, président ; Léon Perrier, Develle, Moxestier, A. Bérard, Lounties, Bedovge, Jugy, Margane Rousseau, FÉret du loncbots, Maiteu, Salles, Dabat, Troté, Buazeix, Charmeil, Garllari, Conte, Monsarrat (représentant M. Dupontem,), Camex, Brithereary, 'Tisserand, Hrtieli, Teissier, Périer de Ferat, Layatm, Bougrayer, Cordier, Rubeyre, Boughtilt, R. Pinot, Petit, Lontie.

Excusés. - MM. Louis Dubors, Arnauné.

M. le Président. - Je donne la parole à M. Dahat, au nom du Ministère de l'Agrìculture.

M. Dльлт. - Voir la note spéciale qui a élé adressée directement aux membres de la Commission. 
M. Le Président. - Nous vous remercions.

La parole est à M. Gaillard, représentant le Ministère de l'Armement.

M. Gaillard, - Vous avez pu constater, Messieurs, que le projet du Ministère de l'Armement diffère essentiellement des autres projets présentés.

Ce projet est provisoire. Il est sommaire. Il ne traite ni de la distinction entre cours d'eau, nj des régimes de concessions, ni de l'institution d'un Office national.

Si le Minislère de l'Armement n'a pas envisagé ces diverses questions, ce n'est pas qu'il s'en désintéresse. Je vais essayer de vous exposer brièvement dans quel état d'esprit a été préparé ce projet de loi. Au moment où cé projet a été établi, il y a deux ou trois mois, quelles que fussent les idées qu'on pouvait avoir sur la durée de la guerre, il semblait peu probable, étant donné l'expéricnce du passé, qu'un projet de loi complet et définitif sur les forces hydrauliques put être voté avant la fin des hostilités. Il faut se féliciter du grand service que rendra la Commission actuellement réunie ici, en abrégeant de beaucoup le temps nécessaire au vote de la loi.

Le Ministère de l'Armement est un ministère provisoire. Né de la gucre, il doit naturellement disparâ̂tre avec elle. Dans ces conditions, pour le Ministère de l'Armement, étudier et préparer un projet de loi définitif, projet de loi destiné à ètre voté et, par conséquent, appliqué après sa disparition, ne lui a pas paru rentrer dans ses attribulions; tout au moins le Ministre de l'Armement ne l'a pas pensé.

Par contre, en ce qui concerne l'établissement de chutes d'eau dans les meilleures conditions possibles et le plus rapidement possible, il a poursuivi cette tâche par l'intermédiaire des Minislères intéressés el je tiens, au sujet de cette collaboration, à remencier les représentants desdits Ministères. Mais, dans la poursuite de cette tâche, il a éprouvé deux sortes de difficultés : difficultés financières d'abord (que M. le Représentant du Sous-Secrétarial d'Etat des Fabrications de guerre vous exposera tout à l'heure) et difficultés relatives aux droits de riveraineté. Je ne veux pas m'étendre sur ce sujet que vous connaissez mieux que moi. Vous savez que les propriétaires riverains exagèrent souvent leurs droits ; quelquefois même, quelles que soient les conditions qu'on leur propose, ils refusent de céder leurs droits de riveraineté. Dans ces conditions, que reste-t-il à faire S'il n'y a pas refus absolu, mais simplement prétentions exagérées, de deux choses l'une : l'industricl les accepte (cela a naturellement une répercussion sur ses frais qui se trouvent majorés, mais enfin, c'est secondaire) on il les refuse et se tourne vers nous en nous demandant de réquisitionner la chute à son profit ; mais la réquisition a des effets passagers, provisoires, qui cesseront avec la guerre, de sorte que l'industriel, non seulament est obligé de prendre l'engagement de se substituer complèlement à l'Etat pour tous les frais qui peuvenl résulter de la réquisition, mais encore, apròs la guerre, l'état de réquisition cessant, il est obligé d'accepter les conditions du proprictaire, quelles qu'elles soient, ou de procéder à la remise en élat. Cette perspective est lerrible : elle représente une responsabilité que beaucoup d'industriels ne veulent pas encourir.

Qu'avons-nous voulu? Nous avons voulu pouvoir éviter ces obstacles au moyen de l'expropriation.

Tel est, en dehors de la question financière dont on vous parlera, le but de notre projet de loi.

Je sais bien qu'on peut faire une objection et même plusieurs, mais une surtout. En échange de cette réquisition, de ces avanlages que nous donnons à l'industriel, nous lui demandons de s'engager à exploiter d'arrès le rógime de la loi future. C'est l'inconnu.

Mais on pourrait bien dire que la concession, que tout le monde est d'accord pour demander, ne sera pas d'une durée inférieure à $7^{5}$ ans, et c'est un des principaux aléas qui disparaît ; ensuite, même en ne précisant pas la durée, l'aléa représenté par l'obligation de se conformer a la concession dont les conditions peuvent être inconnues, est encore moindre que l'aléa représenté par le système de la réquisition. Je crois que, malgré tout, il y a un progrès.

Je voudrais vous parler aussi d'un autre ordre de fails. Je vous ai dit tout à l'heurc que le Ministre de l'Armement, bien qu'il n'ait rien propusé à ce sujel, ne se désintéressait pas dos questions soulevées dars les aulres projets.

Il y en a une qui l'inléresse particulièrement. C'est la création d'un Office national des forces hydrauliques. Le Ministre de l'Armement, un peu avant l'établissement du projet de loi, avait eu l'intention de créer un Office national des forces hydrauliques et il avait pensé à en douner la présidence à un des membres du Parlement ici présent. Mais, il s'est heurté à des difficultés de l'ordre de aclles pour la solution desquolles nous sommes réunis. Après de nombreux pourparlers, la question n'elail pas encore résolue au moment oì M. Albert Thoms est parti pour la Russie,

La création d'un . Office national des forces hydrauliques apporterait une amélioration considérable si nous pouvions lout de suite nous meltre d'accond sur ses attributions et sa composition et sur le Ministère auquel il serail ratlaché. S'il y avait, sur co dernier point, une difficulté, si vous craignez, en quelque sorte, de* préjuger la solution domnéc par le Parlement à la loi définitive, il y aurail une solution que je puis vous indiquer : le Ministère de l'Armement est un Ministère provisoire et je crois qu'une solution acceptable serait de rallacher cel Office national au Ministère de l'Armennent, lonl au moins jusqu'à la fin de la guerre on jusqu'au vote du projel de loi.

Messieurs, notre projet de loi esi modeste. Je ne veux pas vous en parler plus longuement. te me résume el je conclus :

Je crois qu'il est de l'intérêt de la Défense Nationale de voter les dispositions que nous rous demandons. Il est également de l'intérêt de la Défense Nalionale de créer, le plus tồt possible, un Office national des forces hydrauliques qui assure la liaison indispensable entre les différentes administrations et qui concilie les intérêts divergents cl respectables qui existent el qui resteront Loujours divergents et respectables. Je répite que cet Office pourrait, faute de mieux, être ratlaché au Ministère de l'Amement jusqu’à la fin des hostilités.

Je demanderai mainlenant à $M$. le Prisident de vouloir bien donner la parole a M. le Commandant Cahen, du Sous-Secrétariat d'Etat des Fabrication; de Guerre, qui parlera des difficulies financières.

V. Le Présibent. - la parole est a M. ie Commandant Cahen.

Commandant Camen. - M. Je Sons-Secrélaire d'Ltat aux Fabrications de Guerre a ét charge toul spécialement pendant la guerre de poursuivre l'aménagement non pas au point de vue administratif, mais au point de vue pratique, des chutes d'ean on vue de la Défense Nationale, non pas seulement depuis décembre $\mathrm{I}_{9} \mathrm{6}$, date de la création du Ministère de l'Armenent, mais depuis novembre i 915 , spoque à laquelle $M$. Albert Thomas en tant que sous-secrélaire d'Etal de l'Artillerie et des Munitjons, a établi un service spécial dans le but de pousser à la críation des chutes.

Dans celte tâche quotidienne, au cours de ses rupports avec les industriels, le sous-secrélaire d'Etal a roncontré un cértain nombre de difficultés qu'il lui a parı wile de faire connaîlre à la Commission; les unes sont provisoires et dues a l'Etil de guerre; les autres sont d'ordre permanent, subsisieront apris la guerre el se rattachent, par conséquent, non sculement ati projel de loi du Ministère de l'Armenent, mais encore aux projels soumis par les autres Administrations.

Je puis dire tout d'abord que le Ministère de l'Armement n'a pas rencontré de difficullés administratives on plulôt qu'elles ont été rapidement résolues grhee au concours des administralions des Travaux Publics et de l'Agriculture. On a pu donner beaucoup de souplesse aux formalités administratives et, en fait, je crois qu'aucun travail de chute n'a été arrêté par les enquêtes ou les formalités. Te m'associe done aux remerciements exprimés par M. le contrôleur Gaillard, aux deux administrations intéressées.

Les difficultés provisoires rencontrées sont d'ordre gouver- 
nemental, je n'en dirai que quelques mots : ce sont des difficultés de personnel et de matériel.

En ce qui concerne la difficulié de personnel, elle est due en partie à ce lait quón ne peut travailier aux chutes en haute montagne d'une manière permuncnte. Il taul commencer rapidement les travaux à une certaine epoque pentant la belle saison et, par conséquenl, réunir dans un délai très bref́, un personnel nombreux et comportant des spécialistes : bùcherons, terrassiers, mmeurs, elc..., il y a la des dilficultés assez sérieuses.

La seconde dilliculté est relative au malíriel. Il est impossible de construire à lavarice le makriel destiné à laménagemenl. des chutes; il varie avec chaque chule. Or, nous sommes très réduits comme constructeurs de maleriel électro-mécanique. Vous avons élé obligés de recourir aux usines suisses, anglaises et si, actuellement, on arrive oncors à eflectuer les travaux de génie civil, il cst cerlain que le délai d'établissentenı d'une chuce est suburdonné al delui de construction du matériel.

Passons aux difficultés permanentes qui persisteront apiès la guerre : dilficultés provenant des barreurs de chutes et dilticultés tinaneières.

Lin ce qui concerne les barreurs de chutes el surtout les barreur's de droits de passage, $M$. Le contròleur Gaillard nous en a parté ; mais la principale difficulté, c'est la difficulté linancière.

A l'inverse de la plupart des entreprises industrielles qui se dóveloppent au fur et à ruesure que les besoins de leur industrie leur en imposent la nécessité, les entreprises de distribution de houille blanche se caractérisent par ce fait qu'elles sont obligées d'imrnobiliser, dès le débul, la presque totalité des frais de premier établissement. Il est cerlain que c'est là une des causes qui avant la guere a relandé beaucoup l'aménagement de nos chutes d'ean, et qui lail que les promoteurs de celle industrie n'ont pas trouvé toul le concours qu'elle aurait mórité. Cette difficultí est devenue encore plus grande pendant la gucre. Elle a pu être : 28 septembre 1915 .

Cel arlicle autorise à faire des avances aux fournisseurs de la guerre. Malheureusement. la plupart des entreprises de houille blanche, si elles foumissent l'énergie indispensable aux usines de guerre, n'ont pas de marchés directs avec l'Etat. Il y avait donc la une difficulté assez sérieuse qui a élé résolue gràce au voie de la loi sur les avances du 29 janvier 1917 , autorisant des avances, jusqu à concurrence de $x 30$ millions ì faire par les services de l'artilleric et indiquant que les avances pouraient être failes, en ce qui concerne les entreprises de houille blanche, non seulemonl aux titulaires de marchés, mais mène à colles qui fournissent simplement l'énergie aux usines travaillant pour la Défense Nationale. La question des avances est donc résolue.

Mais entre temps, une autre diffouilé s'est prósentée et c'est celle là que vise le derrier alinéa de l'article 1 et l'article 2 du projel du Ministere de l'Armement. Avec la prolongation de la guerre, le cout des travaux des chules a augmenté dans des proportions considérables. Il dépasse actuellement de soo \% le coût d'avant-guerre; les dópenses de maléricl ont mème augmenté daris de plus fortes proportions. Aussi maintenant, les industriels nous declarent qu'ils ne peuvent supporter la lotalité de celte majoration: Ils demandent que l'Elat en prenne une partie à sa charge. Nous nous heurtons done à une nouvelle difficulté, car rous ne pouvons pa: donner de subvention à tous les induslricls qui voudraient équiper des chmes. Nous ne pouvons traiter dans ec bul qu'avec des entreprencurs de chules ayant des marchés direcis avec la gruerre. C'est ce que nous avons fait tout recemment pour réaliser un programme particulièrement imporlant ; la dilficulté a élé résolu: de la manière suivante : les premiers millions' de kilowatts-lienres on les premières tonnes de produle ólectro-melalluggiques stal majoris d'une cerlaine quanlike représentant la part que l'blat prend à l'amortissement d'une parlie de la super-dépense; inais nsus ne pouvons agir ainsi que vis-â-vis des industriels ayant des marchés directs avec l'Etat. C'est celte nouvelle difficulti que le Vinistre de l'Armement cherche à résoudre en demantant, dans l'arlicle a de son projet, l'autorisation de donner une aide financière à loutes les entreprises de chutes.

Messieurs, malgré ces difficuilés et grâce aux deux facteurs que je vieus de vous inciiquer :-simplification des formalités administratives ef système des avances, nous avons pu établir ou mettre en cours d'amenagemenl des chutes produisant une force de 400.000 cheraux environ, mais nous ne pourrons pas aller audelà sans une nouvelle aide linancière.

Cette difficulté subsistera après la guerre; la majoration des prix de premier élablissement sera sans doute moins importante. Nous sommes là dans le domainc de l'hypothèse et il est difficile de chilfrer la majoration qui subsistera ; mais si l'on admet une majoration de 25 à $30 \%$ sur les prix d'avant-guerre, on n'est sans doule pas loin de la réalité.

Comment les entrepreneurs de chutes d'eau qui créeronl de nouvelles chutes, trouveront-ils une compensation à ces majorations de dépenses? Daus l'augmentation du prix de vente 3 II a'y a pas d'augmentation possible pour les distributeurs d'énergie puisque les tarifs sont tixés d'avance ; les entrepremeurs verroni tonc s'ils peuvent supporter la totalité de la majoration. Mai= tis éprouvent déjà assez de aifficultés puisque les meilleures cnitrepprises de houille blanche n'arrivent pas à rémunérer leur capilal avant cinq ou six ans.

D'autre part, pour les entreprises électro-chimiques ét électrométallurgiques, il s'est créé dans lous les pays un nombre considérable d'usines, de nouvelles relations commerciales se sont nouées et il est certain que, pendant un certain temps, il existera un deséquilibre entre la production et la consommation, qui doil plutọt faire prévoir un abaissement des prix de vente.

Aussi, tenant comple de l'expérience de l'avant-guerre el des difficultés rencontrées pendant la guerre, le sous-secrétarial d'Lital des Fabricalions de Cruerre pense que la loi qui sortira des délibérations de la Commission apportera certainement de grandes améliorations par l'unification de direction et des solulions administratives, mais que lion ne poussera réellement à l'aménagenent rapide des chules d'eau, que s'il est prévu en mônte temps l'organisation du crédit. Sans cette organisation de crédit, nous nous heurterons, 'après la guerre, aux mêmes difficultes, grandies encore, qui ont nui au développement des chutes avant la guerre et qui n'ont pu ètre résolues, pendant la guerre; que par le concours financier de l'Etat.

M. re Prúsident. - Je vous remercie.

Je donne maintenant la parole au représentant de la Chambre syndicale des Forces hydrauliques.

M. Cominer. - M. Bougaull fera, au nom de la Chambre syndicale, l'exposé de nos idées, mais je liens à dire quelques mots pour préciser la position de la questıon.

La Chambre syndicale n'a pas étudié un projet de loi ainsi qu'on a pù le dire improprement. Elle ne vient pas en demanderesse. Ce n'est pas du tout dans l'insulfisance législative qu'elle a trouvé les plus grands obstacles : elle les a trouvés dans des questions d'ordre financier, mais je ne veux pas entrer dans. cette question. Je veux seulement indiquer que notre Ghambre syndicale n'a jamais pensé qu'il entrait dans son rôle de préparer des projets de loi, mais elle considère qu'elle peut apporter sa modeste mais loyale collaboration toutes les fois qu'on fait appel à son concours. Elle l'a fail à différentes reprises et notamment. au sujet de la proposition de la loi proposée par MM. Margaine, Bedouce, Deléglise et Mistral. Là Comission des Travaux Publics de la Chambre, qui avait été saisie d'une proposition, a désirć connaître les objections que cette proposition pouvait soulever de la part des industriels. Elle a chargé son rapporteur de sce mettre en rapport avec la Chambre syndicale. C'est ainsi que nous sommes entrés en rapport avoe elle et que nous nous sommes mis à l'étude d'un projet de loi, ou plutôt que nous avons étudié des amendements qui, réunis, ont formé ce qu'on a appelé un projet de loi.

M. Le Président. - Ce sonl, en somme, des contre-propositions au projet Margaine el Bodouce.

M. Condrer. - Parfailement. 
M. Bedouce. - Vos observations avaient d'ailleurs été provoquées.

M. Cornier. - Oui, et nous avons toujours pu constater, lorsque nous sommes entrés en rapports direc's avec les Commissions parlementaires, que nous arrivions à une entente. En ce qui concerne le projet de loi sur les usines du domaine public, nous étions arrivés, après deux ans d'efforls, à un texte transactionnel - où, naturellement, nous avions sacrifié quelques-unes de nos idées, même sur des points importants - qui a été bien près d'aboutir. Il n'a échoué au Sénat que sur un amendemen: qui a été jugé inacceptable. On peut dire qu'à ce moment, la question a été sur le point d'aboutir.

Je vous demánderai, Monsieur le Président, de laisser la parole à H. Bougault qui vous exposera comment nous comprenons la question; car il étudie la législation des chutes depuis vingt ans.

M. le Président. - Nous vous remercions de votre communication intéressante.

La parole est à $M$. Bougault.

M. Bougnuxt. - Messieurs, d'après tont ce qui a été dit jusqu'ici, il semble bien que nous soyons fous d'accord pour proclamer qu'il faut produire, en France, un grand nombre de kilowatts; il faut augmenter notre production nationale pour des raisons que nous connaissons tous, el si l'on pouvait accorder aux entrepreneurs de chutes le pouvoir de développer leur industrie, personne ne serait plus heureux que la Chambre syndicale; car elle comprend dans son sein les premiers industriels qui, en ${ }^{2} 8_{9} 6$ c. r 898 ont su faire courageusement leur œuvre de précurseurs, ont placé leurs premières canalisations, y ont consacré leur temps, leur expérience, leur savoir et, dans le début, peut-être même quelque chose de plus prosaique encore.

Dans ces conditions, que désirons-nous ? Nous voulons que si l'on fait un projet de loi, il constitue une cuvre vraiment pralique ; aussi, avons-nous rapidement répondu à la demande qui nous était faite de présenter nos amendements au projet de loi proposé par MM. Margaine et Bedouce, n'apportant que le concours de notre expérience et, comme on le disait tout à l'heure, nous n'avons jamais demandé une disposition législative nouvelle.

Pour comprendre l'état d'esprit dans lequel ont été présentés ces amendements, voulez-vous me permettre de vous faire un exposé de la législation actuelle ? On veut la modifier : peutclre faut-il bien savoir ce qu'elle est.

Les chutes d'eau peuvent être envisagées sous un triple aspect : $x^{\circ}$ les chutes d'eau auxquelles M. le sénateur Gérand faisait allusion à la dernière séance, les chutes d'eau dites "fondées cn litre ", quel que soit le cours d'eau sur lequel elles sont placées, chutes d'eau qui remontent à la plus haute antiquité, puisqu'elles sont antéricures à l'Edit de 1566 . Ces chutes, Messieurs, je n'en parle... que pour dire que je n'en parlerai plus. Elles ne représentent en effet qu'une puissance assez faible et tous les projets qui se sont succédés ont été d'acicord pour ne pas toucher à ces antiquités essentiellement respectables. Nous ne parlerons donc pas de ces usines concédées en pleine propriété qui remontent à une période antérieure à l'Edit de Moulins mais les deux autres catégories sont autrement plus importantes.

$2^{\circ}$ Certaines rivières sont appelées rivières publiques ou classées, ou floltables et navigables; quel que soit le litre, c'esl la même chose. Depuis l'Edit de Moulins, confirmé par une Ondonnance de Louis XIV de $\mathrm{I} 66_{9}$, loutes les rivieres " portant bateaux ", c'est-à-dire navigables ou " supportant trains de bois ", c'est-à-dire flottables, font partie du domaine public, qui comprend l'eau, le lit, les francs bords, sans parler des servitudes de halage et de marche-pied. C'es! lo domaine public dans tout ce qu'il y a de plus complet, de plus intangible. Sur cas rivières, les chutes d'eau sont peu importantes. C'est à peine si 200.000 kilowalts sont installés et équipés sur ces cours d’eau.

D’abord, pendant un cerlain lemps, l'administration, un peu esclave de la loi de 1898 , a cru ne pouvoir autoriser l'exploitation des chutes sur ces cours d'eau que par voie. de permissions précaires et révocables données par décret. Il n'y. avait pas de traité avec l'Etat. Il y avait une autorisation qui, d'après la jurisprudence du Conseil d'Etat, élait précaire et qui, bien que jouissant d'une certaine solidité, n'était pas un contrat. De sorte que, pendant longtemps, les industriels, effrayés à tort on à raison, n'ont pas trouvé une véritable mine de kilowalts dans les chutes d'eau du domaine public. Ce n'est que plus tard, en I 914, que, pressés par la force des choses, des décrets nouveaux sont sortis, décrets proposés par le Ministre de Travaux Publics qui a fait en cela une exacte appréciation de ses pouvoirs ; car si, en vertu de la loi de $x \& 98$ il ne pouvait donner, par décrels, que des autorisations précaires et révocables, il trouvail, dans la loi du 27 juillel 1870 , un pouvoir de concession pour toutes les lérivations sur toutes espèces de rivières publiques, concessions qui peuvent être données à la condition que les rivières ne soient pas dérivées sur plus de 20 kilomèlres. Par conséquent, nanti de ce texte de loi, il peut donner une concession pour une chute d'eau qui n'est, en somme, qu'un canal qui véhicule de l'eau tombante. Si bien que, le 25 octobre rgr4, la concession élail donnée pour la chute de Beamont-Montenx, le i 6 avril rgr6, pour celle du Drac inférieur el le 2 février rgiz pour celle de la papeterie de Ja Haye-Descartes. C'est toul ce qu'il y a de plus légal ; cela n’a rien de révolutionnaire ef l'Etat s'est considéré comme nanti de lous pouvoirs pour donner ces concessions.

Cela n'empeche pas que ce n'est pas sur les cours d'eau du domaine public qu'il faul chercher un grand nombre de chutes.

Puisque j’ai l'ocrasion de parler des concessions nouvellement accordées, je puis faire quelques rapprochements de dates : li première concession accordée avait élé demandée en rgor : il a fallu 13 ans et demi entre la première ótape et la dernière. La chute du Drac a ćté, comparativement, à une vilesse beaucoup plus rapide; il n'a fallı. que 6 ans pour l'oblenir puisque le lécret rendu en avril rgr6 avait été demandé en rgro. Celle de la papelerie de la Haye-Descartes a été un pen plus rapide parce qu'elle répondail à des besoins de première imporlance. Vous voyez, Messieurs, que si sur celte catégorie, il n'y a pas grand chose de fait, cela est très explicable.

$3^{\circ}$ Nous arrivons à la calégorie d'usines établies sur les cours d'eau non navigables, aux chutes dites quelquefois " du domaine privé " pour les opposèr à celles du domaine public.

On a dit, Messieurs, et même écrit et imprimé, que celte catégorie de chutes était rógie par la loi du 8 avril $8_{9} 8$ sur le régime des eaux. C'est une énorme errenr. Il faut remonter beaucoup plus hant, au Code civil (art. 6/4), pour trouver le statut des rivières non navigables ni flottables. C'est l'art. 6/4 qui a attribué aux riverains et aux riverains seuls l'usage de l'ean. Cela s'explique surtout par me question historique. Avant l'abolition de la féodalité, les rivières non navigables ni flotlables appartenaient aux seicrneurs en pleine propriété. Au moment de la période révolutionnaire, personne ne se préoccupa de dire à quelle partie de la Nation reviendrait la propriété de cette cau. C'est ainsi que, comme on le voit dans les travanx préparatoires du Code civil, les rédacteurs du Code civil se demandèrent à qui il fallait atlribuer cette propriété. L'attribuer à l'Etat, c'était faire, en quelque sorte, ine féodalité à rebours... et puis, il faut indiquer aussi que les seigneurs n'employaient pas de leurs mains l'eau qui était leur propriété. Ils la concédaient à des roturiers qui payaient à cel effet me redevance. Lorsque les seignenrs partis et émigrés, les roturiers restèrent dans leurs monlins, ils oublièrent de payer la redevance et $s e^{\circ}$ considérèrent comme chez eux.

Donc, faire l'altribution à l'Etat, c'était, en quelque sortc, dépouiller les riverains des avantages dont ils jouissaient et... on se contenta de ne rien dire sur le droit de propriété, en concédant l'usage des caux anx riverains seuls.

Voici ce que dit l'art. 6/4: "... des rivières non navigables ni flottables, c'est-à-dire de toutes celles qui ne sont pas comprises dans l'art. $538 \ldots$ le droit d'user de l'ean appartient an riverain ". Au riverain seul : l'Etat n'a rịen à prétendre. Le Code civil a décidé que, s'il y avait des contestations trop vives entre les riverains pour le droit d'usage, c'était les tribunaux civils qui avaient le droit de réglementation et l'a indiqué dans 
l'articlo 645. Le Code civil donne donc le droit de faire un règlemenl d'eau et l'altribution en plein uságe est, en somme, le fief des riverains yuils ont ronservé jusqu’à présent. Si bien que, lorsqu'on veut aménager une chute d'eau, il faut choisir d'abord l'emplacement ef c'est le technicion qui statue pour indiquer ò doivent ilre placés le barrage el la restitution. Lorsque les deux parentheses sont déterminées, alors d'autres, qui ne sont pas des techniciens, réunissent les parenthèses par la série des contrats qui sont obtenus des riverains. II peut y avoir lieu à rextement d'ean judiciaire en verlu de l'article 645 pour régles la question du droit a'eau qui peut itre facilement solulionnée.

Aure ahose, beamoup plus difficile, f'est de faire l'acquisilim des droils de passage sur les terrains ; car on peul se heurler a des volontós obstinées a ne pas vendre. Il y a quelquefois parmi les paysans, et mème parmi ceux qui ne le sont pas, une certaine mentalité qui fail qu’ils préfèrent manquer une bonne aflaire que de la faire an-memes et la voir faire à d'autres. fous lonvons lit des ennemis irréductibles, depuis le barreur qui est to paysin irraisonne jusqu'au barreur - qui n'est pas le moins terrible - représentant la commune, jusqu'au barreur de sentiment que j’ai rencontró une fois, représenté par une demoiselle, proprićtaire convaineue el célibalaire endureie, qui ne voulail pats qu'on passît dans le tréfonds de son champ. Nous avons en ainsi un nombre considérable de démêlés, mais, avec le temps, nous sommes enfin arrivés à avoir le comlenant pous mettre le contenu, à "mettre de l'eau en bouteilles ") suivant ume spirituelle cxpression. Colle tau fait comme le vin, elle s'améliore en vieillissanl car, s'il y a quelques irrégularilés au débul, la ixrestription les fail dispanâle ave le temps.

Donc. Messionrs, on a Iriomphé du barreur. Laissez-moi vous dipe qui un momenl donne, on aurait pu en triompher beacoup plus facilement. Il aurait fallu simplement ajonter à l'art. 644ce qui a déjà été fait an point de vue de l'agriculture - une disposition. Vous n'ignorez pas que la loi de 845 a été faite d̀ une époque où l'agriculture blait considére comme la mantelle nourricière de la France, nis l'on considérait qu'il fallait absolument rendre intensives les irrigations, inlensive la culure des fourrages. On prévoyail done une juste indemnité, fixéa par les tribunaux civils, it varser ain pronrietaire infermediaire situr en!re une source d'eau ou une riverimete ef un fonds à irriguer.

Celle Joi de 18,5 est appliquée iournellement ; elle consiste a créer lil servilude d'aqueduc. moyennant me compensation en argent, pour ne pas perdre une partie de la richesse nationale.

$\mathrm{Or}_{\mathrm{r}}$, la richesse nalionale, c'est actuellement le kilowatt. C'est l'eau qui fombe, plus encoro que l'eau qui irrigue. Il aurait suffe the dire que cette disposition de la loi de is 845 s'appliquait a l'industrie hydraulicue el la question aurail été tranchée rapidoment, par celle généralisation du droil d'aqueduc.

Voila dome pourquoi notre Chambre symdieale est très fiure l'avoir créc, malgré tontes les difficultés, 8oo.noo kilowatts sur les chules d'eau du domaine privé; quand elle a accompli son wuvre, elle est restée propriólaire el cela lui a donné une grande force, pour supporter les mauvais jonrs.

Te pourais peut-dre rappeles le Congres de jgon que quelques-uns ont fail avor nous. A celle époque, l'acétylène subissait the crise truble et malheureusement, beancoup d'industriels avairul ferme leurs usines. D'autres usines étaient des usines de mise au point simplement parce qu'on cherchait de nouveaux procédés l'electrométallureie, si bien qu'on a pu dire assez. méchamment que lo Congriss de la honille blanche avait été le congrès do trìs belles chutes desservant des usines interdites ef des usines lexmés. Les cluules étaient en effel, à re moment. condamnes à une slagnation dipplorable. Cependant. Messieurs, fiamais vous nave ontendu prononeer te mol de faillite on de liquidation. Les aclionnaires de ces industries sont restés pendant des anmées el des ambérs sans foucher un cenlime, mais ils avaicnt confanuce. Pourquoi ? Parce qu'ils ćlaient propriétaires ef qu'ils pouvaienl ficiloment rerourit aux hypothdques et assurer Irur eridil. Rmmarduez que, lorsqu'il faut idomander au public son argent, il faul hui présenter quelque chose qui soit me affaire " de pòre de famille". Nous ne pouvons pas, pour les usines de distribulion d'énergie, promettre un revenu merveilleux ; nous ne pouvons pas promeltre au bout d'un certain temps le taux de 5 ou $6 \%$; ausssi, le placement doit présenter une sécurité absolue pour compenser ce faible taux, il faut que l'entreprise jouisse d'une liberté d'évolution complète; qu'elle puisse atlendre, persuadée que les résultals viendront à coup sùr. Celte sécurité de l'avenir permel de supporter bien des luttes et évite bien des déboires.

On nous parle aujourd'hui d'une autre idée en proposant la concession avéc durée déterminéc. Je ne la discuterai pas pour le moment. On dit que l'Elat doil unifier d'une façon complète, pour se críer des ressources, les chules d'eau du domaine navigable public. Fn lous cas, nous estimons que, pour être féconde, votre ceuvre doit nous laisser la liberlé d'évolution, sans laquelle il n'y a point d'industric.

Je voudrais maintenant demander à $M$. le Présidenl de me donner à nouveau la parole lorsque M. Margaine aura fait son exposé.

M. LE PrÉsident. - Non ; car nous procédons actuellement à des auditions successives et vous ne prourrez reprendre la parole qu'au moment de la discussion. Dites maintenant tout ce que vous voulez dire en prévoyant au besoin l'argument de l'adversaire.

M. Bovtsult. - Je voudrais indiquer seulement l'esprit des amendemenls que nous apportons au projet qui voudrail imposer la concession pour les chutes d'eau, el décréter qu'aucune usine ne pourra êlre faite qu'avec un acte du pouvoir public. Quel sera l'effet ou l'avanlage pour l'industriel ? Aura-t-il dans ce litre nouveau, une nouvelle arme pour attaquer les banreurs ; Je voudrais vous indiquer quelques phrases de l'amendement que nous apportons sur ce point. Il faudrail que l'usinier, pour lc régime de la concession simple, fut nanti d'un décret - trís commun en matière d'agriculture - qui lui permit, moyennani une juste et préalable indemnité, de passer sur les terres avoisinantes. Sans cela, il n'y aura rien d'efficace. Nous aurons uno propriélé lronquée - puisqu'elle aura au maximum une durćc de 60 ans - mais nous n'aurons aucune facilité pour évoluer. Alors, nous disons :

"Les usines concédées auront le droit de placer moyennant une juste et préalable indemnité lours canalisations souterraines dans les propriétés privées, l'indemnité élant déterminée par les tribunaux civils, etc..."

En adoptant ce texte, nous avons pensé qu'il n'était pas nécessaire de faire quelque chose de nouveau. Vous savez tous qu'uno loi a été discutée Irìs complètement au Parlement, par les deux Chambres. Elle ne visail que los chutes d'eau du domaine public, et l'arrêté de concession on d'autorisation devait nantir l'usinier' du droil que j'ai indiqué lout à l'heure, celui d'occuper les propriétés intermédiaires, moyennant. une juste et préalable indemnilé. Nous avons pensé que, puisque deux fois déjà, le Parlement a vait solutionné cetle question, il y aurait sans doute des chances pour que ce lexte déjà volé soil à nouveau adopté.

Nous avons pensé également à la redevance. Jusqu'à présent, nos sociétés n'en payaient pas puisquo, ayant acheté la chute, elles étaient dans leur propriété.

S'il faut une redevance, nous demanderons qu'elle soit proporlionnelle an nombre de kilowatts produits. Si le kilowatt vendu donne un bon rendement, rien de mieux que l'Etat perçoive unc redlevance proportionnelle, mais qu'on n'aille pas jusqu’à donner un droit d'acris complet dans toules les industries de houil]e hanche. Verriez-vous une de ces industries en plein fonctionnement olligée de créer des parts de fondateurs ou des actions et de permeltre à l'Etat de pénétrer dans loules ses affaires ? Il y a dans la redevance un principe nouveau qui peut sé défendre quoi qu'il soit essentiellement nouveau, mais qui doit être absolument limité comme il convient.

En dernier lieu, on a parlé des usines existantes.

J'ai indiqué le régime dans lequel nous vivions : le régime de la pleine propriété. Si, an bout de $7^{5}$ ans, nous ne devons plus vivre que sons le régime de la concession, c'es! la perte d'une chose qui est sacrée : la propriété. J'estime que cela ne peut pas se faire, sans une véritable indemnité. 
Je résume tout en disant que, dans l'intérêt de l'utilisation des chutes d'eau, c'est-à-dire de l'essor national, il faut que l'industrie hydraulique gande une grande facilité. d'évolution.

M. Le Président. - Nous remercions M. Bougault de sa très intéressante et très utile communication.

La séance est levée à miji ( 4 suivre.)

\section{LA COMMISSION DES FORCES HYDRAULIQUES}

La Chambre des Députés a procédé à l'élection des Membres de la Commission chargée d'examiner les projets de loi sur l'utiliation des Forces hyydrauliques. - Ont été élus :

II. de Baudry-d'Asson (Venidée); Bedouce (Hte-Garonne) ; Léon Béluard (Basses-Pyrénées); Bonniard (Hles-Alpes); Antoine Bonket (Savoie) ; Bouctot (Seine-Inférieure) ; Ferdinand BovGàre (Maine-et-Loire); Marcel Cachin (Seine); Eugène Chenal (Aiil); Connevot (Creuse) ; Cromard (Hte-Savoie) ; Dalbiez (Pyućnées-Orientales); De Dron (Loire-Inférieude); Dunandi (Alpes-Maritimes) ; Laurent Eynac (Hte-Loire); Farsant (Saône(4l-Loire) ; Emile Favre (Hte-Savoic) ; Jovelet (Summe) ; Judet (Crense) ; Lecave-Laplagne (Htes-Pyrénées) ; Charles Lebouco (Seilie) ; Limery (Martinique) ; Lerolle (Seine) ; Alfred Le Roy (Aord) ; Margaine (Narne) ; Maurice Mavsoury (Eure-et-Loir); Nistral (Isère); Maurice Spronck (Seine); Mons (Corrèze); Marius Moutet (Rhône); Berland de llun (Marne) ; Parvy (HteVienue) ; Pścindare (Mame) ; Léon Perrier (Isère) ; Puanche (Hles-Alpes) ; Ponted (Ille-él-Vilaine) ; Ferland Rabier (Loired); Raynaud (Charente) ; Ribexre (Hte-Loire); Marcel Sembat Seine); Talon (Aveyron); Valıène (Hte-Vienne); Vibalin (Correze) ; DE Wevnet (Meurthe-et-Moselle).

Dins sa première séance du 5 octobre, la Commission a ćlu sun Bureau qui est ainsi composé :

Président: M. Fornand Rabien ; Vice-présidenls : MM. BÉRARD, Cholard, Paul Ruberre, Boyctot ; Secrétaires : MM. Judet, Bonkel, Lacave-Laplagne, de Mun.

Dans sa deuxième séance du i2 octobre el après une discussion gónérale sur les projets soumis à son examen, elle a élu rapporleur de la. Commission, notre collaboraleur M. Léon Perrier, dinputé de l'Tsère.

\section{ADMINISTRATION ET INDUSTRIE}

\section{PROFESSION DE FOI D'UN FONCTIONNAIRE RETRAITÉ}

"La Houille Blanche " veut bien mouvrir ses colonnes. Je dois à ses lectcurs de leur ouvris mon âme de fonctionnaire retraité ; ce sexa au surplus la maniène la plus suggestive d'expliquer le scnliment qui me pousse à profiter de l'occasion.

Pendanl les vingl demières années de ma carrière administrative, je me suis occupé des questions de houillc blanche en me plaçant plus spécialement, comme c'était non rôle, au point ide vue des Services publics. Pendant dix ans de celte même période, j’ai collaboré avec les Chambres de Commerce du Sud-Est, à la création d'un "Office des Transports "qui, depuis, dans d'autres régions, a fail sonche d'autres Offices semblables. A cetle collaboration, giti II'a procuré de grandes satisfaclions el de précieuses amiliés, je dois de connaîtro un peu les méthodes el les aspirations de la grande industrie moderne.

Je n'ai pas besoin d'insister sur les " cas de conscience " qu'a fait naîlre semblable dualité de fonctions. Dans lo milieu industriel, on aimait assez le fonctionnaire pour ne lui laisser ignorer aucun des méfails de "M. le Burcau ". Dans le milieu administralif, il falliil qu"on l'aimât beaucoup pour ne pas lui lonir rigueur de chercher, à l'occasion, à introduire les mélhorles industrielles dans la défense des intérêts généraux. Puis la guerre est venue rendre plus évidents les dangers de la "Scission " plus pressant l'emploi des moyens propres à lá fiaire disparaitre. - Dans une démocralie telle que la nòtre, les criliques, souvent justes, dont on crible la bureaucratie, devraient contribuer ¿ détruire le mirage d'une "administration-providence ", appliquant avec rapidité des "lois-panacées ". Cependant, c'est plutòt le contraire qui est vrai, à en juger par la quantité de projets de réformes administratives ou législalives, que fonl surgir les critiques et dont on allend le salut.

La guerre, qui bouleverse el détruit lant de choses, semble aux ycux de certains réformaleurs convaincus, justificl des bouleversements et des destructions dans la machine administrative. La sagesse conseille, au contraire, à une heuro où il faut surtoul intensilier le travail de tons, de ne rien bouleverser, do ne rien délruire, d'amóliorer plithò le lravail commun, administralif el industrial, thar des ententes el kes collaborations nowrehes.

Libéré de mes doubles fonclions, mais non des fóllexions qu'eldes ont pr.voutées, j'éprouve, comme une angoissanl. obsession, lo désir de travailler à fane disparailue fsé malentendus, les barrieres, les cloisons élanches, qui antmvent l'aménagement des foreses liydrauliques.

Je ne change pas de prinl de vore. la défenes de l'intérèt général reste mon seul objectif ; mais, en malière de houllle blanche, comme en toule autre, J'intérèt génólal n'est que la résultante des intérêts parliculicrs el collectifs, ou pour parler avec plus de précision, des intérêts combinés des producleurs ot des consommateurs d'énergie. En s'orgunisanl pour aider l'Administralion à dégager colle résullanle, con sommatcars ef producterrs frrond l'oeure la plus utile, pour leur plus grand profit et pour celui du Pays.

Dans le nouveau litre de "La Houlie blanche ", se glisse un mot gui vaut à lui seul un programme. Pour liner le meilleur parti de l' "énergie hydraudigtue ", on se propose d'en " coordonner " l'emploi avec colui du charbon. Go qu'il faut aussi coordonner, ce sont les efforts de lous ceux qui sont imféresés à la grande cuvre de l'aménagement des eaux : fonctionnaires of industricts, productours et consommaleurs, particuliers, sciéćs of colledivités. Cette "condination " nécossaire doil ètre réalisée, an poinl de vue administralif, par les organismes prévus an Tilac V1 du nouveau projel de loi ; ello sea ulitoment complété, au point nti vue industriel el collectir, par des "Offices d'énergie "rógionaux, analogues aux offices des Transporls.

Comme, par dessis loul, a l'heure actuelle, il faul agir et réaliser, cost par des róalisalions successives que le syslème de la coordination libre marquera, en se développanl, chacune de ses ćlapes. La plus grande cause des relards se trouve inconlestablencul dans l'impuissance de l'Administration à choisir, entro plusieurs compéliteurs d'unc chul" d'eau, "le meillont" ". Pour sortir d'embarras, elle lew dil : entendez-rous. Le systemo de la coordinalion repond à re conseil, qu'il élargit cependant un peu en faisant intervenir dans l'entente les ntilisaleurs de l'óncrgie en général, el plus spécialement les collectivilés chargées des Services Publics, tribulaires de l'énergie.

C'est dans cel esprit de coordinalion, d' "union sacrée ", pour employer l'expression de guerre qui devta survivre à la girfyre, qu'a été rédigé, sur la demande de l'Association française pour le dévoloppement des Travaux publics, lo rapport "La Houille Blanche el la Guerre " ; c'ost dans co même espril que lesoussigné examinera, au point de vue des Services publics, le projet de loi récemment élaboré et que, dans 'une série d'autres études, s'inspiranil d'exemples 